\title{
Mappings of Bounded Distortion Between Complex Manifolds
}

\author{
Min Dai and Xiaokui Yang
}

\begin{abstract}
We obtain Liouville type theorems for holomorphic mappings with bounded $s$-distortion between $\mathbb{C}^{n}$ and positively curved Kähler manifolds.
\end{abstract}

Keywords: bounded distortion, Ricci curvature, Liouville theorem.

\section{Introduction}

The classic Liouville theorem states that- every bounded holomorphic function on the entire complex plane is constant. H.Grötzsch observed that the classic Liouville theorem can be extended to quasi-conformal mappings. A smooth mapping $f: \mathbb{R}^{n} \longrightarrow \mathbb{R}^{n}$ is called quasi-conformal, if it is orientation preserving, and locally it is a diffeomorphism such that

$$
|d f|^{n} \leq K J(f), \quad \text { a.e. }
$$

for some positive constant $K \geq 1$, where $|d f|$ is the operator norm of the Jacobian matrix $d f$ and $J(f)$ is the determinant of $d f$. It is well-known that every holomorphic mapping $f: \mathbb{C} \longrightarrow \mathbb{C}$ is quasi-conformal with $K=1$. More 
precisely, $|d f|^{2}=J(f)$. However, in higher dimensional case, holomorphic mappings are not necessarily quasi-conformal. For example, the holomorphic mapping $f: D=\left\{\left(z_{1}, z_{2}\right) \in \mathbb{C}^{2}|| z_{2} \mid>\frac{1}{2}\right\} \longrightarrow \mathbb{C}^{2}$ with $f\left(z_{1}, z_{2}\right)=\left(z_{1}, z_{2}^{2}\right)$ is not quasiconformal, but it satisfies $|d f|^{2}=J(f)$. Here $2<\operatorname{dim}_{\mathbb{R}} \mathbb{C}^{2}=4$. For more details, one can see Example 2.2 and Example 2.14. Hence, we can consider smooth mappings with bounded $s$-distortion,

$$
|d f|^{s} \leq K J(f)
$$

for some $s \in(0, \infty)$.

In this paper, we consider holomorphic mappings with bounded $s$-distortion between complex manifolds and obtain Liouville type theorem for such mappings. Let's recall several classic Liouville type theorem for holomorphic mappings and quasi-conformal mappings between manifolds.

Theorem 1.1 (Yau's Schwarz Lemma). Let $M$ be a complete Kähler manifold with Ricci curvature bounded from below by $K_{1}$. Let $N$ be another Hermitian manifold with holomorphic bisectional curvature bounded from above by a negative constant $K_{2}$. Then if there is a non-constant holomorphic mapping f from $M$ into $N$, we have $K_{1} \leq 0$ and

$$
f^{*}\left(d S_{N}^{2}\right) \leq \frac{K_{1}}{K_{2}} d S_{M}^{2}
$$

In particular, if $K_{1} \geq 0$, every holomorphic mapping from $M$ into $N$ is constant.

For more details about Schwarz Lemma and related Liouville type theorem, we refer the reader to Ahlfors([1]), Yau([20]), Kobayashi([14]), Chen-Yang([4]), Tossati([19]) and reference therein.

The study of the Schwarz Lemma and Liouville type theorem for non-holomorphic quasi-conformal(quasi-regular) mapping was started from Kiernan([12]) in our knowledge. From then, there are many mathematicians study the harmonic mappings with various bounded distortion, for example, Chern, Goldberg, Har'El, Ishihara, Petridis, Shen([3],[6], [7], [8],[9],[17]), etc. We summarize their works wildly in the following:

Theorem 1.2 (Generalized Schwarz Lemma). Let $M, N$ be complete Riemannian manifolds. Suppose the Ricci curvature of $M$ is bounded below by $-K_{1}$ and the 
sectional curvature of $N$ is bounded from above by $-K_{2}$ where $K_{1}, K_{2}>0$. If $f: M \longrightarrow N$ is a harmonic $K$-quasi-regular mapping, then

$$
f^{*}\left(d s_{N}^{2}\right) \leq C \frac{K_{1}}{K_{2}} d s_{M}^{2}
$$

where $C$ is a positive constant depending on $K$ and the dimension of the manifolds. In particular, if $f: \mathbb{R}^{m} \longrightarrow N$ is a harmonic $K$-quasi-regular mapping, then $f$ is a constant.

The common conditions in the Schwarz Lemma and Liouville type theorem are

(1) The target should be negatively curved;

(2) The mapping should satisfy certain bounded distortion condition.

In this paper, we consider the Liouville type theorem for positively curved targets instead of negatively curved ones. By a geometric interpretation of inequality (1.2), we obtain:

Main Theorem Let $(N, h)$ be a complete Kähler manifold of complex dimension $n$, and $f:\left(\mathbb{C}^{n}, \omega_{\mathbb{C}^{n}}\right) \longrightarrow(N, h)$ be holomorphic. If $f$ is a mapping with bounded $2 s$-distortion and $N$ has the curvature property $\left(Q_{s}\right)$, then $f$ is constant.

In fact, the curvature condition $\left(Q_{s}\right)$ has a geometric explanation. It is equivalent to the Griffiths positivity of the (formal) vector bundle $G=T^{*} N \otimes K_{N}^{* 1 / s}$. For any compact Kähler manifold with $c_{1}(M)>0$, the anti-canonical line bundle $K_{N}^{*}$ is a positive line bundle, so there exists some small $s \in(0, \infty)$ such that the vector bundle $G$ is Griffiths positive, i.e., the curvature condition $Q_{s}$ can be satisfied automatically(Theorem 2.10).

Corollary 1.3. Let $M$ be a compact Kähler manifold with $c_{1}(M)>0$. Then there exist a Kähler metric $\omega$ and some $s_{0} \in(0, \infty)$ such that any holomorphic mapping $f: \mathbb{C}^{n} \longrightarrow(M, \omega)$ with bounded s-distortion, $s \in\left(0, s_{0}\right)$, is constant.

In particular, for $\mathbb{P}^{n}$, we obtain 
Corollary 1.4. If $f: \mathbb{C}^{n} \longrightarrow \mathbb{P}^{n}$ is a holomorphic mapping with bounded $s$ distortion, $0<s<n+1$, with respect to the canonical metrics, then $f$ is a constant.

There do exist holomorphic mappings of bounded $s$-distortion between $\mathbb{C}^{n}$ and $\mathbb{P}^{n}$ for some $s$. For example, the canonical map $f:\left(\mathbb{C}^{n}, \omega_{\mathbb{C}^{n}}\right) \longrightarrow\left(\mathbb{P}^{n}, \omega_{F S}\right)$

$$
f\left(z_{1}, \cdots, z_{n}\right)=\left[1, z_{1}, \cdots, z_{n}\right]
$$

is a holomorphic mapping with bounded $(2 n+2)$-distortion and it fails to be a mapping of bounded $s$-distortion for any $s \in(0,2 n+2)$. In particular, it is not quasiconformal.

Acknowledgements. The second author is very grateful to Valentino Tosatti for some useful discussions and suggestions.

\section{Mappings of bounded $s$-distortion between mani- folds}

In the paper [18], the authors consider a generalized version of mappings with bounded distortion.

Definition 2.1. A smooth mapping $f: \Omega \subset \mathbb{R}^{n} \longrightarrow \mathbb{R}^{n}$ has bounded $s$-distortion $(0<s<\infty)$ if it is a constant or a local diffeomorphism and

$$
|d f|^{s} \leq K J(f), \quad \text { for } \quad x \in \mathbb{R}^{n}
$$

for some positive constant $K$.

It is obvious that, mappings of bounded $n$-distortion are quasiconformal. But in general, a holomorphic mapping can be bounded $s$-distortion for some $s$ but not quasiconformal.

Example 2.2. If $f: \mathbb{C}^{2} \longrightarrow \mathbb{C}^{2}, f\left(z_{1}, z_{2}\right)=\left(z_{1}, z_{2}^{2}\right)$ and $z_{1}=x+\sqrt{-1} y, z_{2}=$ $s+\sqrt{-1} t$, then $(d f)^{t} \cdot d f$ is a $4 \times 4$ diagonal matrix with diagonal entries $1,1,4\left(s^{2}+\right.$ $\left.t^{2}\right), 4\left(s^{2}+t^{2}\right)$. Therefore

$$
J(f)=4\left(s^{2}+t^{2}\right), \quad|d f|=\max \left\{1,2 \sqrt{s^{2}+t^{2}}\right\}
$$


There is no positive constant $K$ such that

$$
|d f|^{4} \leq K J(f)
$$

holds on $\mathbb{C}^{2}$. In fact, if $s^{2}+t^{2}>1$,

$$
\frac{|d f|^{4}}{J(f)}=4\left(s^{2}+t^{2}\right)
$$

which is undounded on $\mathbb{C}^{2}$. However, on $D=\left\{\left(z_{1}, z_{2}\right) \in \mathbb{C}^{n}|| z_{2} \mid>\frac{1}{2}\right\}$,

$$
\frac{|d f|^{2}}{J(f)}=1
$$

Hence, $f: D \longrightarrow \mathbb{C}^{2}, f\left(z_{1}, z_{2}\right)=\left(z_{1}, z_{2}^{2}\right)$ is a holomorphic mapping of bounded 2-distortion, but it is not a mapping of bounded 4-distortion.

Now we go to define mappings of bounded distortion between manifolds. Let $f:(M, g) \longrightarrow(N, h)$ be a smooth mapping between oriented $n$-dimensional Riemannian manifolds. In the local coordinates $\left(x^{\alpha}\right)$ and $\left(y^{i}\right)$ on $M$ and $N$ respectively, we set

$$
J(x, f)=\frac{f^{*} d v_{h}}{d v_{g}}=\sqrt{\frac{\operatorname{det}\left(h_{i j}\right)(f(x))}{\operatorname{det}\left(g_{\alpha \beta}\right)(x)}} \operatorname{det}\left(\frac{\partial f^{i}}{\partial x^{\alpha}}\right)
$$

where $f^{i}=y^{i} \circ f$. The pointwise operator norm of $d f$ with respect to the metrics $g$ and $h$ is given by

$$
|d f(x)|^{2}=\max _{X \neq 0} \frac{\left|f_{*} X\right|_{h}^{2}}{|X|_{g}^{2}}=\max _{X \neq 0} \frac{\sum_{i, j, \alpha, \beta} h_{i j} f_{\alpha}^{i} f_{\beta}^{j} X^{\alpha} X^{\beta}}{\sum_{\alpha, \beta} g_{\alpha \beta} X^{\alpha} X^{\beta}}
$$

where $f_{\alpha}^{i}=\frac{\partial f^{i}}{\partial x^{\alpha}}$ and $X=X^{\alpha} \frac{\partial}{\partial x^{\alpha}}$. Here and henceforth we sometimes adopt the Einstein convention for summation. It is obvious that $|d f(x)|^{2}$ is the maximal eigenvalue of the positive definite matrix $A=\left(A_{\alpha \beta}\right)$ with respect to the metric $g$ where $A_{\alpha \beta}=\sum_{i, j} h_{i j} f_{\alpha}^{i} f_{\beta}^{j}$. So $J(x, f)$ and $|d f(x)|$ are well defined and do not depend on the local coordinates. 
Definition 2.3. A smooth mapping $f:(M, g) \longrightarrow(N, h)$ between oriented $n$ dimensional manifolds is said to have bounded $s$-distortion $(0<s<\infty)$ with respect to the metrics $g$ and $h$ if it is a constant or a local diffeomorphism with

$$
|d f(x)|^{s} \leq K J(x, f)
$$

for some positive constant $K$.

Now we recall some notations on Kähler manifolds. Let $\left\{z^{\alpha}\right\}_{\alpha=1}^{n}$ be the local holomorphic coordinates on the Kähler manifold $(M, g)$, then the metric $g$ is locally represented by a Hermitian positive matrix $\left(g_{\alpha \bar{\beta}}\right)$, that is

$$
g_{\alpha \bar{\beta}}=g\left(\frac{\partial}{\partial z^{\alpha}}, \frac{\partial}{\partial z^{\beta}}\right)
$$

If $\nabla$ is the complexified Levi-Civita connection on $M$, the curvature of $\nabla$ is locally given by

$$
R_{\alpha \bar{\beta} \gamma \bar{\delta}}=-\frac{\partial^{2} g_{\alpha \bar{\beta}}}{\partial z^{\gamma} \partial \bar{z}^{\delta}}+g^{\lambda \bar{\mu}} \frac{\partial g_{\alpha \bar{\mu}}}{\partial z^{\gamma}} \frac{\partial g_{\lambda \bar{\beta}}}{\partial \bar{z}^{\delta}}
$$

The Ricci curvature of $\nabla$ is

$$
\operatorname{Ric}(g)=\frac{\sqrt{-1}}{2} R_{\alpha \bar{\beta}} d z^{\alpha} \wedge d \bar{z}^{\beta}
$$

where

$$
R_{\alpha \bar{\beta}}=g^{\gamma \bar{\delta}} R_{\alpha \bar{\beta} \gamma \bar{\delta}}=-\frac{\partial^{2} \log \operatorname{det}\left(g_{\lambda \bar{\mu}}\right)}{\partial z^{\alpha} \partial \bar{z}^{\beta}}
$$

For more basic notations of complex geometry, we refer the reader to the book $[5]$.

Let $f:(M, g) \longrightarrow(N, h)$ be a holomorphic mapping between Kähler manifolds with complex dimension $n$. In the local holomorphic coordinates $w^{\alpha}$ and $z^{i}$ on $M$ and $N$ respectively,

$$
d f=f_{\alpha}^{i} d w^{\alpha} \otimes \frac{\partial}{\partial z^{i}} \in \Gamma\left(M, T^{1,0} M \otimes f^{*}\left(T^{1,0} N\right)\right)
$$

where $f^{i}=z^{i} \circ f$ and $f_{\alpha}^{i}=\frac{\partial f^{i}}{\partial w^{\alpha}}$ since $f$ is holomorphic. The operator norm $|d f|^{2}$ is

$$
|d f|^{2}=\max _{X \neq 0} \frac{\left|f_{*} X\right|_{h}^{2}}{|X|_{g}^{2}}=\max _{X \neq 0} \frac{\sum_{i, j, \alpha, \beta} h_{i \bar{j}} f_{\alpha}^{i} \bar{f}_{\beta}^{j} X^{\alpha} \bar{X}^{\beta}}{\sum_{\alpha, \beta} g_{\alpha \bar{\beta}} X^{\alpha} \bar{X}^{\beta}}
$$


for any $X=X^{\alpha} \frac{\partial}{\partial w^{\alpha}} \in \Gamma\left(M, T^{1,0} M\right)$. On the other hand, the Riemannian volume and the Kähler metric is related by

$$
d V=\frac{\omega^{n}}{n !}
$$

therefore by formula (2.2),

$$
J(f)=\frac{f^{*} \omega_{h}^{n}}{\omega_{g}^{n}}=\frac{\operatorname{det}\left(h_{i \bar{j}}\right)}{\operatorname{det}\left(g_{\alpha \bar{\beta}}\right)}\left|\operatorname{det}\left(f_{\alpha}^{i}\right)\right|^{2}
$$

The real dimension of the manifolds $M$ and $N$ is $2 n$. That is, a bounded $2 n$ distortion mapping is quasiconformal.

The curvatures of manifolds and $J(f)$ are related by the following formula(see $[2])$.

Lemma 2.4. Let $(M, g)$ and $(N, h)$ be two complete Kähler manifolds of complex dimension $n$. Let $f:(M, g) \longrightarrow(N, h)$ be a holomorphic mapping, then at a point $J(f) \neq 0$, the following formula holds

$$
\operatorname{Ric}\left(\omega_{g}\right)-f^{*} \operatorname{Ric}\left(\omega_{h}\right)=\frac{\sqrt{-1}}{2} \partial \bar{\partial} \log J(f)
$$

Proof. If $\varphi$ is a holomorphic function without zero point,

$$
\partial \bar{\partial} \log |\varphi|^{2}=\frac{|\varphi|^{2} \partial \varphi \wedge \overline{\partial \varphi}-\bar{\varphi} \partial \varphi \wedge \varphi \overline{\partial \varphi}}{|\varphi|^{4}}=0
$$

Since $\operatorname{det}\left(f_{\alpha}^{i}\right)$ is holomorphic, by formula (2.7), we obtain

$$
\begin{aligned}
\frac{\sqrt{-1}}{2} \partial \bar{\partial} \log J(f) & =\frac{\sqrt{-1}}{2} \partial \bar{\partial} \log \frac{\operatorname{det}\left(h_{i \bar{j}}\right)}{\operatorname{det}\left(g_{\alpha \bar{\beta}}\right)} \\
& =\operatorname{Ric}\left(\omega_{g}\right)-f^{*}\left(\operatorname{Ric}\left(\omega_{h}\right)\right)
\end{aligned}
$$

Lemma 2.5. Let $\left(N, h, \omega_{h}\right)$ be a complete Kähler manifold. If $X$ is a holomorphic vector field of $N$, then on a small neighborhood of point $P$ such that $X_{P} \neq 0$,

$$
T=\sqrt{-1}\left(\frac{\partial^{2} \log |X|_{h}^{2}}{\partial z^{k} \partial \bar{z}^{l}}+\sum_{i, j} R_{i \bar{j} k \bar{l}} \frac{X^{i} \bar{X}^{j}}{|X|_{h}^{2}}\right) d z^{k} \wedge d \bar{z}^{\ell}
$$


is a semi-positive $(1,1)$ form where $R_{i \bar{j} k \bar{l}}$ is the curvature of $(N, h)$ given by

$$
R_{i \bar{j} k \bar{l}}=-\frac{\partial h_{i \bar{j}}}{\partial z^{k} \partial \bar{z}^{l}}+h^{s \bar{t}} \frac{\partial h_{i \bar{t}}}{\partial z^{k}} \frac{\partial h_{s \bar{j}}}{\partial \bar{z}^{l}}
$$

Proof. We can choose the normal coordinates $\left(z^{1}, \cdots, z^{n}\right)$ centered at the fixed point $P \in N$, i.e., $h_{i \bar{j}}(P)=\delta_{i \bar{j}}$ and $\frac{\partial h_{i \bar{j}}}{\partial z^{k}}(P)=\frac{\partial h_{i \bar{j}}}{\partial \bar{z}^{l}}(P)=0$. More precisely, on the small neighborhood of $P$,

$$
h_{i \bar{j}}(z)=\delta_{i \bar{j}}-R_{i \bar{j} k \bar{l}}(P) z^{k} \bar{z}^{l}+O\left(|z|^{3}\right)
$$

Now we assume $X=X^{i} \frac{\partial}{\partial z^{i}},|X|_{h}^{2}=\sum_{i, j} h_{i j} X^{i} \bar{X}^{j}$. At point $P$,

$$
\partial \bar{\partial}|X|_{h}^{2}=\sum_{i, j, k, l}\left(\frac{\partial^{2} h_{i \bar{j}}}{\partial z^{k} \partial \bar{z}^{l}} X^{i} \bar{X}^{j}+h_{i \bar{j}} \frac{\partial X^{i}}{\partial z^{k}} \frac{\overline{\partial X^{j}}}{\partial z^{l}}\right) d z^{k} \wedge d \bar{z}^{l}
$$

that is,

$$
\frac{\partial^{2}|X|_{h}^{2}}{\partial z^{k} \partial \bar{z}^{l}}=-\sum_{i, j} R_{i \bar{j} k \bar{l}} X^{i} \bar{X}^{j}+\sum_{i} \frac{\partial X^{i}}{\partial z^{k}} \frac{\overline{\partial X^{i}}}{\partial z^{l}}
$$

At the fixed point $P$,

$\frac{\partial^{2} \log |X|_{h}^{2}}{\partial z^{k} \partial \bar{z}^{l}}=\frac{-\sum_{i, j} R_{i \bar{j} k \bar{l}} X^{i} \bar{X}^{j}}{|X|_{h}^{2}}+\frac{|X|_{h}^{2}\left(\sum_{i} \frac{\partial X^{i}}{\partial z^{k}} \overline{\frac{\partial X^{i}}{\partial z^{l}}}\right)-\left(\sum_{j} X^{j} \frac{\partial \bar{X}^{j}}{\partial \bar{z}^{l}}\right)\left(\sum_{i} \bar{X}^{i} \frac{\partial X^{i}}{\partial z^{k}}\right)}{|X|_{h}^{4}}$

If we set

$$
T_{k \bar{l}}=\frac{\partial^{2} \log |X|_{h}^{2}}{\partial z^{k} \partial \bar{z}^{l}}+\sum_{i, j} R_{i \bar{j} k \bar{l}} \frac{X^{i} \bar{X}^{j}}{|X|_{h}^{2}}
$$

then by Schwarz inequality,

$$
\sum_{k, l} T_{k \bar{l}} v_{k} \bar{v}_{l}=\frac{|X|_{h}^{2}\left(\sum_{i} \frac{\partial X^{i}}{\partial z^{k}} v_{k} \frac{\partial X^{i}}{\partial z^{l}} v_{l}\right)-\left(\sum_{j} X^{j} \frac{\partial \bar{X}^{j}}{\partial \bar{z}^{l}} \bar{v}_{l}\right)\left(\sum_{i} \bar{X}^{i} \frac{\partial X^{i}}{\partial z^{k}} v_{k}\right)}{|X|_{h}^{4}} \geq 0
$$

for any $v \in \mathbb{C}^{n}$. 
Definition 2.6. Let $(N, h)$ be a complete Kähler manifold. We say that $(N, h)$ has the curvature property $\left(Q_{s}\right)$ if for any $u, v \in \mathbb{C}^{n}-\{0\}$,

$$
\frac{1}{s} \sum_{i, j, k, \ell} R_{i \bar{j}} h_{k \bar{\ell}} u^{i} \bar{u}^{j} v^{k} \bar{v}^{\ell}-\sum_{i, j, k, \ell} R_{i \bar{j} k l} u^{i} \bar{u}^{j} v^{k} \bar{v}^{\ell}>0
$$

for some constant $s \in(0, \infty)$.

Remark 2.7. We have a geometric explanation of the curvature formula (2.10). If $E$ and $F$ are two holomorphic vector bundles with connections $\nabla^{E}$ and $\nabla^{F}$, then the curvature of the induced connection on $E \otimes F$ is

$$
R=R^{E} \otimes I d_{F}+I d_{E} \otimes R^{F}
$$

where $R^{E}$ and $R^{F}$ are the curvatures of $E$ and $F$ respectively. Apply this to the (formal) vector bundle

$$
G=T^{*} N \otimes K_{N}^{* 1 / s}
$$

we get the curvature of it, which is locally given by

$$
\widehat{R}_{i \bar{j} k \bar{l}}=\frac{1}{s} R_{i \bar{j}} h_{k \bar{l}}-R_{i \bar{j} k \bar{l}}
$$

where $K_{N}$ is the canonical line bundle of the manifold $N$. The positivity condition in the definition is nothing but the Griffiths positivity of the vector bundle $G$, i.e.

$$
\widehat{R}_{i \bar{j} k \bar{l}} u^{i} \bar{u}^{j} v^{k} \bar{v}^{\ell}>0
$$

Example 2.8. Let $\left(\mathbb{P}^{n}, \omega_{F S}\right)$ be the complex projective space with the FubiniStudy metric. Locally, it can be written as

$$
\omega_{F S}=\frac{\sqrt{-1}}{2} g_{i \bar{j}} d z^{i} \wedge d \bar{z}^{j}
$$

It is well-known that

$$
R_{i \bar{j} k \bar{l}}=g_{i \bar{j}} g_{k \bar{l}}+g_{i \bar{l}} g_{k \bar{j}}, \quad R_{i \bar{j}}=g^{k \bar{\ell}} R_{i \bar{j} k \bar{\ell}}=(n+1) g_{i \bar{j}}
$$

It is obvious that, for nonzero $X$,

$$
\frac{1}{s} R_{i \bar{j}}|X|_{g}^{2}-R_{i \bar{j} k \bar{l}} X^{k} \bar{X}^{l}=\frac{n+1-s}{s}|X|_{g}^{2} g_{i \bar{j}}-g_{i \bar{l}} g_{k \bar{j}} X^{k} \bar{X}^{l}
$$

Then basic linear algebra shows that the above matrix is Hermitian positive if and only if

$$
\frac{n+1-s}{s}-1>0 \Longleftrightarrow 0<s<\frac{n+1}{2}
$$


Corollary 2.9. $\left(\mathbb{P}^{n}, \omega\right)$ has the property $\left(Q_{s}\right)$ with $0<s<\frac{n+1}{2}$.

Formally, we have

$$
T^{*} \mathbb{P}^{n} \otimes\left(K_{\mathbb{P}^{n}}^{*}\right)^{\frac{1}{s}}=T^{*} \mathbb{P}^{n} \otimes \mathcal{O}_{\mathbb{P}^{n}}\left(\frac{n+1}{s}\right)
$$

which is positive if and only if $\frac{n+1}{s}>2$.

More generally, we have

Theorem 2.10. If $M$ is a compact Kähler manifold with $c_{1}(M)>0$, then there exist a Kähler metric $\omega$ and some $s_{0} \in(0, \infty)$ such that $(M, \omega)$ satisfies the curvature condition $\left(Q_{s}\right)$ for any $s \in\left(0, s_{0}\right)$.

Proof. By Yau's solution of Calabi conjecture $([21])$, if $c_{1}(M)>0$, there exists a Kähler metric such that

$$
\operatorname{Ric}(\omega)>0
$$

Since the manifold is compact, there exists $\varepsilon>0$ such that

$$
\operatorname{Ric}(\omega) \geq \varepsilon \omega
$$

It is obvious that the holomorphic bisectional curvature $R_{i \bar{j} k \bar{l}}$ is also bounded, that is, there exists $\varepsilon_{1}>0$ such that

$$
R_{i \bar{j} k \bar{l}} X^{k} \bar{X}^{l} \xi^{i} \bar{\xi}^{j} \leq \varepsilon_{1}|X|_{\omega}^{2}|\xi|_{\omega}^{2}
$$

Hence, there exists $s_{0} \in(0, \infty)$ such that

$$
\frac{|X|_{\omega}^{2}}{s_{0}} R_{i \bar{j}} \xi^{i} \bar{\xi}^{j}-R_{i \bar{j} k \bar{l}} X^{k} \bar{X}^{l} \xi^{i} \bar{\xi}^{j} \geq \frac{\varepsilon-s_{0} \varepsilon_{1}}{s_{0}}|X|_{\omega}^{2}|\xi|_{\omega}^{2}>0
$$

for any nonzero $X=\sum X^{i} \frac{\partial}{\partial z^{i}}$ and $\xi=\sum \xi^{j} \frac{\partial}{\partial z^{j}}$. The constant $s_{0}$ depends on the manifold $M$ and $\omega$.

Theorem 2.11. Let $(N, h)$ be a complete Kähler manifold of complex dimension $n$, and $f:\left(\mathbb{C}^{n}, \omega_{\mathbb{C}^{n}}\right) \longrightarrow(N, h)$ be holomorphic. If $f$ is a mapping with bounded $2 s$-distortion and $N$ has the property $\left(Q_{s}\right)$, then $f$ is constant. 
Proof. Let $\omega_{h}$ be the corresponding Kähler form of $h$. On the local holomorphic coordinates $\left(z^{1}, \cdots, z^{n}\right)$ of $N$, we can write

$$
\omega_{h}=\frac{\sqrt{-1}}{2} \sum_{i, j} h_{i j} d z^{i} \wedge d \bar{z}^{j}
$$

If $f$ is not constant, then for any nontrivial constant holomorphic vector field $Y=Y^{\alpha} \frac{\partial}{\partial w^{\alpha}}$ on $\mathbb{C}^{n},\left|f_{*} Y\right|_{h}^{2}$ is nonzero everywhere by the local diffeomorphism property of mappings of bounded distortion. We claim that

$$
L=\frac{\sqrt{-1}}{2} L_{\gamma \bar{\delta}} d w^{\gamma} \wedge d \bar{w}^{\delta}:=\frac{\sqrt{-1}}{2} \partial \bar{\partial} \log \left|f_{*} Y\right|^{2}+\frac{1}{s} f^{*}\left(\operatorname{Ric}\left(\omega_{h}\right)\right)
$$

is a semi-positive $(1,1)$-form on $\mathbb{C}^{n}$ if $N$ has curvature property $\left(Q_{s}\right)$.

In fact, we have

$$
f_{*} Y=\sum_{i, \alpha} \frac{\partial f^{i}}{\partial w^{\alpha}} Y^{\alpha} \frac{\partial}{\partial z^{i}}, \quad\left|f_{*} Y\right|_{h}^{2}=\sum_{i, j, \alpha, \beta} h_{i \bar{j}}(f) \frac{\partial f^{i}}{\partial w^{\alpha}} \frac{\overline{\partial f^{j}}}{\partial w^{\beta}} Y^{\alpha} \bar{Y}^{\beta}
$$

By a similar computation as Lemma 2.5,

$$
\frac{\partial^{2} \log \left|f_{*} Y\right|^{2}}{\partial w^{\alpha} \partial \bar{w}^{\beta}}=-R_{i \bar{j} k \bar{\ell}} \cdot \frac{\partial f^{k}}{\partial w^{\gamma}} \frac{\overline{\partial f^{\ell}}}{\partial w^{\delta}} \cdot \frac{\frac{\partial f^{i}}{\partial w^{\alpha}} \frac{\overline{\partial f^{j}}}{\partial w^{\beta}} Y^{\alpha} \bar{Y}^{\beta}}{\left|f_{*} Y\right|_{h}^{2}}+W_{\gamma \bar{\delta}}
$$

where $\left(W_{\gamma \bar{\delta}}\right)$ is a semi-positive Hermitian matrix by Schwarz inequality. In the sense of Hermitian positivity, we obtain

$$
L_{\gamma \bar{\delta}} \geq \frac{1}{s} R_{i \bar{j}} \frac{\partial f^{i}}{\partial w^{\gamma}} \frac{\partial f^{j}}{\partial w^{\delta}}-R_{i \bar{j} k \bar{\ell}} \frac{\partial f^{k}}{\partial w^{\gamma}} \frac{\partial f^{\ell}}{\partial w^{\delta}} \frac{\frac{\partial f^{i}}{\partial w^{\alpha}} \overline{\frac{\partial f^{j}}{\partial w^{\beta}}} Y^{\alpha} \bar{Y}^{\beta}}{\left|f_{*} Y\right|_{h}^{2}} \geq 0
$$

where the last step follows by the the curvature condition $\left(Q_{s}\right)$.

On the other hand, by Lemma 2.4,

$$
\frac{1}{s} f^{*}\left(\operatorname{Ric}\left(\omega_{h}\right)\right)=\frac{1}{s} \operatorname{Ric}\left(\omega_{\mathbb{C}^{n}}\right)-\frac{\sqrt{-1}}{2 s} \partial \bar{\partial} \log J(f)=-\frac{\sqrt{-1}}{2} \partial \bar{\partial} \log J(f)^{\frac{1}{s}}
$$

By formula (2.11), we obtain

$$
L=\frac{\sqrt{-1}}{2} \partial \bar{\partial} \log \left(\frac{\left|f_{*} Y\right|_{h}^{2}}{J(f)^{\frac{1}{s}}}\right) \geq 0
$$


The weight function

$$
\Phi=\frac{\left|f_{*} Y\right|_{h}^{2}}{J(f)^{\frac{1}{s}}}
$$

is plurisubharmonic on $\mathbb{C}^{n}$. By the definition (2.6) of $|d f|$,

$$
\left|f_{*} Y\right|_{h}^{2} \leq|d f|^{2}|Y|_{g}^{2}
$$

Then we get

$$
\Phi \leq \frac{|d f|^{2}|Y|_{g}^{2}}{J^{\frac{1}{s}}(f)} \leq K^{\frac{1}{s}}|Y|_{g}^{2}=K^{\frac{1}{s}} \sum_{\alpha}\left|Y^{\alpha}\right|^{2}
$$

for some positive constant $K$ by the definition of bounded $2 s$-distortion mapping. Since $Y$ is a constant vector field on $\mathbb{C}^{n}$, we obtain that $\Phi$ is a plurisubharmonic function bounded from above and so $\Phi$ is constant on $\mathbb{C}^{n}$. That is $L=0$. By formula (2.13), we know

$$
\frac{\partial f^{i}}{\partial w^{\gamma}}=0
$$

for any $i$ and $\gamma$. Finally, we obtain $f$ is anti-holomorphic, and so it is constant.

Corollary 2.12. Let $M$ be a compact Kähler manifold with $c_{1}(M)>0$. Then there exist a Kähler metric $\omega$ and some $s_{0} \in(0, \infty)$ such that any holomorphic mapping $f: \mathbb{C}^{n} \longrightarrow(M, \omega)$ with bounded s-distortion, $s \in\left(0, s_{0}\right)$, is constant.

In particular, for $\mathbb{P}^{n}$ we obtain

Corollary 2.13. If $f: \mathbb{C}^{n} \longrightarrow \mathbb{P}^{n}$ is a holomorphic mapping with bounded $s$ distortion, $0<s<n+1$, with respect to the canonical metrics, then $f$ is a constant.

Proof. It follows easily from Corollary 2.9 .

Example 2.14. Let $f:\left(\mathbb{C}^{n}, \omega_{\mathbb{C}^{n}}\right) \longrightarrow\left(\mathbb{P}^{n}, \omega_{F S}\right)$ be the canonical map

$$
f\left(z_{1}, \cdots, z_{n}\right)=\left[1, z_{1}, \cdots, z_{n}\right]
$$

Then $f$ is a holomorphic mapping with bounded $(2 n+2)$-distortion and it fails to be a mapping of bounded $s$-distortion for any $s \in(0,2 n+2)$. In particular, it is not quasiconformal. 
In fact, on the chart $U_{0}=\left\{\left[z_{0}, \cdots, z_{n}\right] \mid z_{0}=1\right\}$,

$$
\omega_{F S}=\frac{\sqrt{-1}}{2} \partial \bar{\partial} \log \left(1+\sum_{i=1}^{n}\left|z_{i}\right|^{2}\right)=\frac{\sqrt{-1}}{2} \cdot h_{i \bar{j}} \cdot d z_{i} \wedge d \bar{z}_{j}
$$

where

$$
h_{i \bar{j}}=\frac{\left(1+\sum\left|z_{i}\right|^{2}\right) \delta_{i j}-\bar{z}_{i} z_{j}}{\left(1+\sum\left|z_{i}\right|^{2}\right)^{2}}
$$

It is obvious that $\left(h_{i \bar{j}}\right)$ has two different eigenvalues, $\lambda_{\max }=\left(1+\sum\left|z_{i}\right|^{2}\right)^{-1}$ with multiplicity $(n-1)$ and $\lambda_{\min }=\left(1+\sum\left|z_{i}\right|^{2}\right)^{-2}$ with multiplicity 1 . Therefore,

$$
\operatorname{det}\left(h_{i \bar{j}}\right)=\left(1+\sum_{i=1}^{n}\left|z_{i}\right|^{2}\right)^{-(n+1)}
$$

By formula (2.7),

$$
J(f)=\frac{f^{*}\left(\omega_{F S}^{n}\right)}{\omega_{\mathbb{C}^{n}}^{n}}=\left(1+\sum_{i=1}^{n}\left|z_{i}\right|^{2}\right)^{-(n+1)}
$$

On the other hand, on the chart $U_{0}, f\left(z_{1}, \cdots, z_{n}\right)=\left(z_{1}, \cdots, z_{n}\right)$ and $d f=I$. By formula (2.6), $|d f|^{2}$ is the maximal eigenvalue $\lambda_{\max }=\left(1+\sum_{i=1}^{n}\left|z_{i}\right|\right)^{-1}$ of the Hermitian positive matrix $\left(h_{i \bar{j}}\right)$. Therefore

$$
|d f|=\left(1+\sum_{i=1}^{n}\left|z_{i}^{2}\right|\right)^{-\frac{1}{2}}
$$

By the expression of $J(f)$ and $|d f|$, we obtain

$$
|d f|^{2(n+1)}=J(f)
$$

For any $0<s<2(n+1)$, the ratio

$$
\frac{|d f|^{s}}{J(f)}=\left(1+\sum_{i=1}^{n}\left|z_{i}\right|\right)^{n+1-\frac{s}{2}}
$$

is unbounded on $\mathbb{C}^{n}$. In summary, $f: \mathbb{C}^{n} \longrightarrow \mathbb{P}^{n}$ is a holomorphic mapping of bounded $(2 n+2)$-distortion and it fails to be a mapping of bounded $s$-distortion for any $s \in(0,2 n+2)$. 


\section{References}

[1] Ahlfors, L. An extension of Schwarz's lemma. Trans. Amer. Math. Soc. 43 (1938), no. 3, 359-364.

[2] Chern, S. S. On the holomorphic mappings of hermitian manifolds of the same dimension, Proc. Symp. Pure Math, Vol. 11, 1968

[3] Chern, S. S.; Goldberg, S. I. On the volume decreasing property of a class of real harmonic mappings. Amer. J. Math. 97 (1975), 133-147.

[4] Chen, Z. H; Yang, H. C. A class of Liouville theorems. (Chinese) Acta Math. Sinica 28 (1985), no. 2, 218-232.

[5] Demailly, J. P. Complex analytic and algebraic geometry, book on line http://www-fourier.ujf-grenoble.fr/ demailly/books.html

[6] Goldberg, S. I.; Har'El, Z. Mappings of almost Hermitian manifolds. J. Differential Geom. 14 (1979), no. 1, 67-80 (1980).

[7] Goldberg, S. I.; Ishihara, T. Harmonic quasiconformal mappings of Riemannian manifolds. Amer. J. Math. 98 (1976), no. 1, 225-240

[8] Goldberg, S. I.; Ishihara, T. Petridis, N. C. Mappings of bounded dilatation of Riemannian manifolds. J. Differential Geometry 10 (1975), no. 4, 619-630

[9] Goldberg, S. I.; Har'El, Z. A general Schwarz lemma for Riemannianmanifolds. Bull. Soc. Math. Grce (N.S.) 18 (1977), no. 1, 141-148.

[10] Iwaniec, T.; Martin, G. Geometric function theory and non-linear analysis. Oxford Mathematical Monographs. The Clarendon Press, Oxford University Press, New York, 2001. xvi+552.

[11] Iwaniec, T. p-harmonic tensors and quasiregular mappings. Ann. of Math. (2) 136 (1992), no. 3, 589-624.

[12] Kiernan, P. J. Quasiconformal mappings and Schwarz's lemma. Trans. Amer. Math. Soc. 1481970 185-197 
[13] Kobayashi, S. Volume elements, holomorphic mappings and the schwarz lemma, Proc. Symp. Pure Math, Vol. 11,1968

[14] Kobayashi, S. Volume elements, holomorphic mappings and Schwarz's lemma. 1968 Entire Functions and Related Parts of Analysis (Proc. Sympos. Pure Math., LaJolla, Calif., 1966) pp. 253-260 Amer. Math. Soc., Providence, R.I.

[15] Marden, A.; Rickman, S. Holomorphic mappings of bounded distortion. Proc. Amer. Math. Soc. 46 (1974), 226-228

[16] Rickman, S. Quasiregular mappings. Ergebnisse der Mathematik und ihrer Grenzgebiete (3), 26. Springer-Verlag, Berlin, 1993. x+213 pp

[17] Shen, C. L. A generalization of the Schwarz-Ahlfors lemma to the theory of harmonic maps. J. Reine Angew. Math. 348 (1984), 23-33.

[18] Troyanov, M.; Vodop'yanov, S. Liouville type theorems for mappings with bounded (co)-distortion. Ann. Inst. Fourier (Grenoble) 52 (2002), no. 6, $1753-1784$.

[19] Tossati, V. A general Schwarz lemma for almost-Hermitian manifolds. Comm. Anal. Geom. 15 (2007), no. 5, 1063-1086.

[20] Yau, S. T. A general Schwarz lemma for Kähler manifolds. Amer. J. Math. 100 (1978), no. 1, 197-203.

[21] Yau, S. T. On the Ricci curvature of a compact Kähler manifold and the complex Monge-Ampére equation. I. Comm. Pure Appl. Math. 31 (1978), no. $3,339-411$

Min Dai

School of Computer \& Computing Science

Zhejiang University City College

Hangzhou 310015, China

E-mail: hadriana@163.com 
Xiaokui Yang

Department of Mathematics

University of California at Los Angeles

Los Angeles, CA 90095-1555

E-mail: xkyang@math.ucla.edu 